\title{
Antibiotic Use, Disposal and Awareness of Human Health Risk Associated With Consuming Antibiotics in Groundwater Among People Living in Informal Settlements of Kisumu, Kenya
}

Kellen Joyce Karimi ( $\sim$ karimikellen@gmail.com )

University of the Witwatersrand and University of Nairobi

\section{Aijaz A.}

University of the Witwatersrand

\section{Duse A.G.}

University of the Witwatersrand

Mwanthi A. M.

University of Nairobi

Ayah R.

University of Nairobi

\section{Research article}

Keywords: human health effects, antibiotic use, antibiotic disposal, groundwater use, informal settlements

Posted Date: July 21st, 2020

DOI: https://doi.org/10.21203/rs.3.rs-42198/v1

License: (c) (i) This work is licensed under a Creative Commons Attribution 4.0 International License.

Read Full License 
1 ANTIBIOTIC USE, DISPOSAL AND AWARENESS OF HUMAN HEALTH RISK 2 ASSOCIATED WITH CONSUMING ANTIBIOTICS IN GROUNDWATER AMONG 3 PEOPLE LIVING IN INFORMAL SETTLEMENTS OF KISUMU, KENYA

4 Author, Karimi J. K. ${ }^{1,2}$, Aijaz A. ${ }^{1}$, and Duse A.G., ${ }^{1}$ Mwanthi A. M., ${ }^{2}$ Ayah R. ${ }^{2}$

$5{ }^{1}$ Department of Clinical Microbiology \& Infectious Diseases, School of Pathology, Faculty of

$6 \quad$ Health Sciences, University of the Witwatersrand.

$7 \quad{ }^{2}$ School of Public Health, College of Health Sciences, University of Nairobi.

$9 \quad$ June 2020

\section{Abstract}

Background: Informal settlements across SSA have proliferated with increased urban population.

Characterized by inadequate water supply, inhabitants resort to groundwater for domestic use. This happens oblivious of water contamination with emerging contaminants in form of antibiotics and their derivatives. Development of antibiotic resistant genes and antibiotic resistant bacteria, causes ill health, thus interventions that increase level of awareness of health effects of consuming water contaminated with antibiotics are requisite. This involves protecting human, animal and environmental in totality in a One-Health approach. This study sought to determine antibiotic use, disposal and level of awareness of health effects associated with consuming water contaminated with antibiotics.

Methods: A cross-sectional study of a random sample of 447 households in selected informal settlements of Kisumu County, Kenya was conducted in September 2019. A structured questionnaire was generated based on research questions and administered to heads of households

24 to assess, antibiotic use, disposal and level of awareness of health effects associated with 25 consuming water contaminated with antibiotics. Data was entered and analyzed in SPSS version 26 20. 
27 Results: Level of awareness of health effects of consuming water contaminated with antibiotics

28 was low, 35\% $(n=156)$, especially among households where a member had used an antibiotic

29 within one month prior to the time of study; $\mathrm{p}=0.03$. Groundwater was used by $99.8 \%(\mathrm{n}=446)$ for

30 various purposes, including drinking $(9 \%(n=40))$, cooking $(18 \%(n=43))$, washing utensils $(79 \%$

$31(\mathrm{n}=353))$, clothes $(96 \%(427))$ and house (95\% (421)). About half the households reported

32 antibiotic use $43 \%(n=193)$. Among this, $74 \%(n=144)$ consulted a health worker in a healthcare

33 facility for prescription. Respondents who had taken antibiotics did not always complete doses but

34 reported to have kept the remainder for next time they would get sick (54\%). About 32\% disposed

35 remainder of antibiotics in pit latrines, compost pits (10\%) while $4 \%$ reported to have burnt them.

36 Conclusion and recommendation: Groundwater is the major water source for domestic use in

37 informal settlements. With low awareness of risks associated with consuming water contaminated with antibiotics, a looming public health concern due to antibiotic resistance necessitate attention

39 to avert health effects that may lead to ill health.

40

41 Key words: human health effects, antibiotic use, antibiotic disposal, groundwater use, informal 42 settlements

\section{Introduction}

46 Antibiotics are antimicrobial drugs used to kill or prevent bacteria growth and there are various

47 classes of antibiotics used in humans and animals [1][2]. Antibiotic use is limited to persons

48 confirmed to be suffering from a given infection and also is prescribed by a health worker in a

49 health facility. Antibiotics have been classified as an emerging source of contaminants which need

50 attention to prevent negative consequences to non-targeted humans, animals and ecosystems [3].

51 They can get into the environment through a various channels of entry including through; hospital 
52 waste disposal, wastewater, sewage, animal waste and pit latrines adjacent groundwater sources $53[4][5][6]$.

55 Proliferation of informal settlements in cities in the SSA had led to increase need for housing [4].

56 This upsurge has led to increased demand for safe water for domestic use and sanitation [4]. While

57 the quantity and quality of groundwater continue to be explored as source to supplement surface

58 water sources used in most cities and towns, increased population and therefore human activity

59 may lead to its contamination with a myriad of pollutants which include antibiotics [7][8].

60

61 Antibiotics in the environments are a public health risk and an emerging global threat to health

62 systems due to rising antibiotic resistance [9]. Health effects of exposure to antibiotics have been

63 documented in marine environments but involuntary exposure among humans in any other

64 environment that they may have day to day direct contact with [3]. Effect on microbes treated

65 using the same antibiotics found in the environment have been found to be resistance [1], which

66 results to inability to treat infections due to development of resistance, making research in the area

67 is significant. Knowledge of health effects as a result of exposure to antibiotics through drinking

68 water is limited in the general population [10]. Prioritizing human health should be synchronized

69 with protection of animal health, as well as that of the environment since a complex interaction

70 among the three occur in an interaction otherwise referred to as the One-Health triad [11]. A One-

71 Health approach [12] to achieve optimal health for the humans, animals and the environment is key

72 in addressing the looming global antibiotic resistance burden. This paper aims to explore antibiotic

73 use and disposal among households as well as the level of awareness of health effects as a result

74 of consuming water that is contaminated with antibiotics is also assessed among people living in

75 informal settlements. 


\section{$77 \quad$ Materials and Methods}

78 A cross-sectional study of people living in selected informal settlements of Kisumu County in

79 Kenya was conducted in the month of September 2019. The informal settlements were selected 80 purposively since they fall within the mapped water points within the AFRIWATSAN project 81 site[13]. The sample of households to be included into the study was randomly selected and 82 determined based on the population[14] of each informal settlement. Proportionate allocation of 83 households was employed to achieve the calculated sample size of 442 households. From each 84 household, one head was selected to be included in the study and interviewed upon giving informed 85 consent.

86 Households were randomly selected around mapped water points which acted as reference points 87 and are within the AFRIWATSAN project site[13]. Every $5^{\text {th }}$ household was selected and included 88 in the sample until the determined sample size [15] of 442 households was achieved. The 442 89 households were proportionately distributed among five (5) informal settlements in the study area, namely Manyatta A \&B, Nyalenda A\&B and Obunga, based on the total populations in each of

91 the settlements. A structured questionnaire was administered to 448 household heads. Antibiotic 92 use, disposal and awareness of health effects associated with consuming water that is contaminated 93 with antibiotics was sought among the household heads. Data were entered in IBM* SPSS* 94 Statistics version 20 and checked for errors. The dataset was cleaned and analyzed. Descriptive 95 statistics were used to summarize continuous variables whereas relationships/ associations in 96 categorical variables were assessed using chi-square tests.

\section{$98 \quad$ Results}


99 A total of 447 of households were visited in the five informal settlements. The number of 100 households in each informal settlement were as follows: Obunga 24 (5.4\%), Manyatta A 143

101 (32.1\%), Manyatta B 89 (19.9\%), Nyalenda A 96 (21.4\%) and Nyalenda B (21.2\%) There were 102 more female $(75 \%(n=337))$ respondents than males, majority of whom were below the age of 45 103 years $(79 \%(n=354))$.

104 Ground water use

105 Almost all $(99.8 \% \mathrm{n}=446)$ of the respondents used groundwater sources. Groundwater was used 106 for various purposes in the households, including drinking $(9 \%(n=40))$, cooking $(18 \%(n=43))$, 107 washing utensils $(79 \%(\mathrm{n}=353))$, clothes $(96 \%(427))$ and house $(95 \%(421))$. Respondents 108 reported to have used the groundwater sources for a period of time that extended to 32 years (94\%). 109 Others reported to have found the water in use since they were born and could have been in use 110 for more than 32 years.

111 Antibiotic use

112 Antibiotic use among households in the past one month before the day of the interview was 113 reported to be $43 \%(n=193)$. Antibiotics were taken following a recommendation from friends $11426 \%(n=13)$ and $76 \%(n=36)$ used antibiotics based on a previous experience. Among this 115 consuming antibiotics, $26 \%(\mathrm{n}=50)$ did not consult a doctor for the antibiotics prescription. The 116 level of awareness of health effects of consuming water that is contaminated with antibiotics was 117 found to be $35 \%(\mathrm{n}=158)$, more so in households where antibiotic use was reported $\mathrm{p}=0.003$. There 118 was however no significant association between awareness of health effects associated with 119 consuming antibiotic in water and groundwater use.

120

\section{Discussion}


122 Antibiotic use among persons living in informal settlements of Kisumu County was found to be at

$12343 \%$. The antibiotics were either prescribed by a healthcare worker in a health facility, bought

124 based on previous experience or advice from a friend. A prescription from qualified medical

125 personnel is required for anyone to get access to antibiotics. Predictors of antibiotic use and misuse

126 in a given family have been prescribed by [16] as perceived behavioral control, social norms,

127 interaction between attitudes, beliefs and knowledge as well as the presence of a healthcare

128 practitioner in the household. Self-medication using antibiotics is a factor in development of drug

129 resistance, more often the practice is due to convenience of the user, leading to misuse of 130 antibiotics [17]. The Ministry of Health acknowledges lack of information on antibiotic use and/or 131 misuse in the population [10]. To this effect, the ministry indicates that a robust data collection 132 system on surveillance on antibiotic use and awareness of health effects as a result of exposure to 133 the antibiotics using a One-Health approach.

135 An estimated $60 \%$ of Kisumu residents live in the informal settlements, and suffer inadequacies 136 like lack of water and proper sanitation facilities [14]. Contamination of groundwater sources have 137 been found to be higher in areas where population density is high [18]. Antibiotics get into 138 groundwater through various ways that include direct dumping with garbage, excreted through 139 feces and urine, from hospital and industrial waste[1][19] and some of the antibiotics have been 140 detected in water [20]. Some respondents reported to have kept the remainder of antibiotics not 141 utilized for future use whereas others disposed in a pit latrine, compost pit or burned.

143 Antibiotics pose a threat to human and animal health as the effect as a result of involuntary 144 exposure are not clear. This interaction of the humans, animals in the environment comprise a 145 complex interact otherwise referred to as the One-Health triad [11]. Any pollutant that may be 
146 present in the environment require attention to ensure protection of the components of both humans 147 and animals.

148 Level of awareness on the health effects of consuming water that is contaminated with antibiotics 149 was low (43\%) among residents of the informal settlements of Kisumu County. Antibiotic use in 150 Kenya has not been quantified making it difficult to implement guidelines [10] on use and 151 resistance. The situational analysis further recommends intervention by increasing awareness of 152 antibiotic use through public forums that target the providers and consumers of antibiotics. 153 Conversely, an assessment of awareness of human health risk associated with antibiotic use among 154 livestock keepers in Tanzania and knowledge of the antibiotic resistance concept show revealed 155 that farmers were not aware of antibiotic resistance [21]. Some farmers did not know diseases 156 treatable with antibiotics and that antibiotic use among livestock had health effects among humans. 157 Customers in a pharmacy in Norway reported a high level of knowledge of antibiotic resistance 158 [22]. There were however, knowledge gaps on the type of infections that are treatable with 159 antibiotics, recommending campaigns to increase level of knowledge among persons in 160 occupations who are not familiar with the health field [22].

\section{Conclusion and recommendations}

165 This study explored antibiotic use, disposal and level of awareness of health effects associated 166 with consuming water contaminated with antibiotics among people living in informal settlements. 167 Level of awareness of health effects was generally low even with the continued use of antibiotics, 168 bringing to perspective efforts by the World Health Organization and government of Kenya the 169 effectiveness of the awareness creation through the 'World Antibiotic Awareness week. This is an 
170 annual event aimed at improving awareness and understanding of antimicrobial resistance and

171 strengthen knowledge through surveillance and research among antibiotic providers and users. To

172 effectively provide safe water for this population that is free of contamination especially with

173 antibiotics, efforts should be geared towards awareness creation and implementation of the Global

174 Action Plan that guarantees prevention and treatment of infections with safe and effective 175 antibiotics which is couples with safe disposal of the antibiotics to reduce environmental 176 contamination which results to antibiotic resistance. The realization of this goal is possible in a 177 multidisciplinary teams instituted in the One-Health Approach.

178

179 Declarations

180 Abbreviations

181 ARG: Antibiotic Resistant Genes, ARB: Antibiotic Resistant Bacteria, MoH: Ministry of Health:

182 AMR: Antimicrobial Resistance, SSA: Sub Sahara Africa

\section{Acknowledgements}

184 We would like to appreciate the heads of households in the informal settlements in Kisumu County 185 i.e. (Obunga, Manyatta A \&B, and Nyalenda A\&B) for giving consent to conduct the study. We 186 are also indebted by the dedication of the research assistants led by Mr. Francis Omondi. We also 187 appreciate Janet Musia for her input in data entry and management.

\section{Author's contributions}

189 KJK was involved in conception of the study, data collection, entry and analysis and interpretation 190 of the results and drafting of the manuscript. AA, AD, MM and RA were involved in the study 
191 design, close study supervision and in the revision of the manuscript. All authors read and 192 approved the final manuscript.

\section{$193 \quad$ Funding}

194 This research is supported by the Consortium for Advanced Research Training in Africa (CARTA). 195 CARTA is jointly led by the African Population and Health Research Centre and the University of 196 the Witwatersrand and funded by the Carnegie Corporation of New York (Grant No--B 8606.R02), 197 Sida (Grant No:54100029), the DELTAS Africa Initiative (Grant No: 107768/Z/15/Z). The DELTAS 198 Africa Initiative is an independent funding scheme of the African Academy of Sciences (AAS)'s 199 Alliance for Accelerating Excellence in Science in Africa (AESA) and supported by the New 200 Partnership for Africa's Development Planning and Coordinating Agency (NEPAD Agency) with 201 funding from the Wellcome Trust (UK) (Grant No: 107768/Z/15/Z) and the UK government. The 202 research is also supported by the Africa Water Sanitation (AFRIWATSAN) project funded by the 203 Royal Society Capacity Building Initiative and the UK Department for International Development 204 (DFID).

205 Availability of data and materials

206 All data related to this study will be made available upon request.

\section{Ethics approval and consent to participate}

209 Ethical clearance to conduct research was obtained from three institutions as follows; the Health

210 Research Ethic Committee of the university of the Witwatersrand (HREC. Protocol Number

211 M190412); the Kenyatta National Hospital and University of Nairobi Ethics and Research 
212 Committee (KNH/UoN-ERC. Ref No. P71910/2018); and the National Commission for Science,

213 Technology and Innovation (Ref No. NACOSTI/P/19/3232/28732).

214 Consent to participate in the study was obtained from the study participants before the interview

215 was conducted. The study participants signed an informed consent form.

\section{Consent for publication}

217 Not applicable

218 Competing interests

219 The authors declare no competing interests.

220 Author details

$221{ }^{1}$ Department of Clinical Microbiology and Infectious Diseases, School of Pathology, Faculty of 222 Health Sciences, University of the Witwatersrand, 1 Jan Smuts Avenue, Braamfontein 2000,

223 Johannesburg, South Africa. ${ }^{2}$ School of Public Health, College of Health Sciences, University of 224 Nairobi P.O. Box 19676-00202, Kenyatta National Hospital, Along Mbagathi Road Off Ngong 225 Road, Nairobi, Kenya.

\section{References}

230 [1] M. Danner, A. Robertson, V. Behrends, and J. Reiss, "Science of the Total Environment Antibiotic pollution in surface fresh waters : Occurrence and effects," Sci. Total Environ., vol. 664, pp. 793-804, 2019. 
233 [2] I. T. Carvalho and L. Santos, "Antibiotics in the aquatic environments : A review of the European scenario," Environ. Int., vol. 94, pp. 736-757, 2016.

[3] V. Corbel et al., "Evidence for inhibition of cholinesterases in insect and mammalian nervous systems by the insect repellent deet.," BMC Biol., vol. 7, no. 1, p. 47, 2009.

[4] E. W. Kimani-Murage and A. M. Ngindu, "Quality of water the slum dwellers use: The case of a Kenyan slum," J. Urban Heal., vol. 84, no. 6, pp. 829-838, 2007. near the municipal solid waste landfill," Sci. Total Environ., vol. 609, pp. 966-973, 2017.

S. Matongo, G. Birungi, B. Moodley, and P. Ndungu, "Pharmaceutical residues in water and sediment of Msunduzi River, KwaZulu-Natal, South Africa," Chemosphere, 2015.

[7] M. S. U. Rehman, N. Rashid, M. Ashfaq, A. Saif, N. Ahmad, and J. I. Han, "Global risk of pharmaceutical contamination from highly populated developing countries,"

Chemosphere, vol. 138. pp. 1045-1055, 2015.

8] J. Okotto-okotto, L. Okotto, H. Price, S. Pedley, and J. Wright, “A Longitudinal Study of Long-Term Change in Contamination Hazards and Shallow Well Quality in Two Neighbourhoods of," vol. 2014, pp. 4275-4291, 2015.

[9] M. Ferri et al., "Antimicrobial resistance : A global emerging threat to public health systems," Crit. Rev. Food Sci. Nutr., vol. 57, no. 13, pp. 2857-2876, 2017.

[10] Global Antibiotic Resistance Parnership, "Situation Analysis and Recommendations: Antibiotic Use and Resistance in Kenya. Washington, DC and New Delhi: Center for Disease Dynamics, Economics \& Policy," 2011.

[11] M. F. Davis et al., "Checklist for One Health Epidemiological Reporting of Evidence (COHERE)," One Heal., vol. 4, no. January, pp. 14-21, 2017. 
OF ANTIMICROBIAL RESISTANCE, Kenya,” no. April, p. 7,13, 2017.

258

[13] J. R. Kanoti, D. Olago, N. Opiyo, C. Nyamai, S. Dulo, and R. Ayah, "Microbial and Physical Chemical Indicators of Groundwater Contamination in Kenya: A Case Study of Kisumu Aquifer System, Kenya,” J. Water Resour. Prot., vol. 11, no. 04, pp. 404-418, 2019.

[14] M. Maoulidi, "Kisumu Millenium Development Goals Multi-Sector Household Survey," no. December, pp. 1-46, 2012.

[15] M. A. Pourhoseingholi, M. Vahedi, and M. Rahimzadeh, "Sample size calculation in medical studies," vol. 6, no. 1, pp. 14-17, 2013.

[16] M. K. Byrne et al., "The drivers of antibiotic use and misuse : the development and investigation of a theory driven community measure," pp. 1-11, 2019.

[17] A. Rajendran, K. Kulirankal, P. Rakesh, and S. George, "Prevalence and pattern of antibiotic self-medication practice in an urban population of Kerala, India: A crosssectional study," Indian J. Community Med., vol. 44, no. 5, pp. 42-45, Oct. 2019.

[18] E. Ngumba, A. Gachanja, and T. Tuhkanen, Occurrence of selected antibiotics and antiretroviral drugs in Nairobi River Basin, Kenya, vol. 539. 2015.

[19] J. P. Graham and M. L. Polizzotto, "Review Pit Latrines and Their Impacts on Groundwater Quality: A Systematic Review,”vol. 121, no. 5, pp. 521-530, 2013.

[20] E. Sanganyado and W. Gwenzi, "Science of the Total Environment Antibiotic resistance in drinking water systems : Occurrence, removal, and human health risks," Sci. Total Environ., vol. 669, pp. 785-797, 2019.

[21] A. Katakweba, M. M. A. Mtambo, J. E. Olsen, and A. Muhairwa, "Awareness of human health risks associated with the use of antibiotics among livestock keepers and factors that contribute to selection of antibiotic resistance bacteria within livestock in Tanzania," 
Livest. Res. Rural Dev., vol. 24, Oct. 2012.

282 [22] M. Waaseth et al., "Knowledge of antibiotics and antibiotic resistance among Norwegian pharmacy customers - A cross-sectional study," BMC Public Health, vol. 19, no. 1, pp. 1$12,2019$.

285 